\title{
Intrathecal synthesis in particular types of multiple sclerosis
}

\author{
Vladimira Sladkova ${ }^{\mathrm{a}}$, Jan Mares ${ }^{\mathrm{a}}$, Petr Hlustik ${ }^{\mathrm{a}}$, Jana Langova ${ }^{\mathrm{b}}$, Petr Kanovsky ${ }^{\mathrm{a}}$
}

\begin{abstract}
Aim. The aim of this study was to determine whether there were any differences in intrathecal synthesis of immunoglobulin $\mathrm{G}(\mathrm{lgG})$ (lgG index) and number of oligoclonal bands (OCB) among particular types of multiple sclerosis (MS). Methods. 120 cerebrospinal fluid (CSF) samples were examined from 29 clinically isolated syndrome (CIS) patients and 91 MS patients (77 patients with relapsing-remitting MS (RR), 6 patients with primary progressive course of the disease (PP) and 8 patients in secondary progression (SP)); mean age $=42$ years (range $=18$ to 70 years). Albumin and IgG in serum and CSF was evaluated using nephelometry; an albumin quotient (CSF albumin/serum albumin), an lgG quotient (CSF IgG/serum IgG) and an IgG index (lgG quotient / albumin quotient) were then calculated. OCB were assessed using isoelectric focusing (IEF) on agarose gel, followed by immunoblotting. All patients were evaluated using the Kurtzke Expanded Disability Status Scale (EDSS).
\end{abstract}

Results. No statistically significant differences between the IgG index and OC bands relative to particular types of MS were found. Further, there were no significant correlations between EDSS values and intrathecal synthesis (IgG index: QlgG / Qalbumin) and OC bands.

Conclusion. No difference in intrathecal synthesis (IgG index) and the number of OCB between different types of MS was confirmed.

Key words: multiple sclerosis, cerebrospinal fluid, oligoclonal bands

Received: September 14, 2011; Accepted with revision: May 9, 2012; Available online: June 1, 2012 http://dx.doi.org/10.5507/bp.2012.054

${ }^{a}$ Department of Neurology, University Hospital Olomouc, Czech Republic

${ }^{b}$ Department of Biostastistics, University Hospital Olomouc

Corresponding author:Vladimira Sladkova, e-mail: sladkovav@centrum.cz

\section{INTRODUCTION}

Multiple sclerosis (MS) is an inflammatory autoimmune disease of the central nervous system (CNS), directed against the myelin sheath, leading to demyelination and axonal loss. The inflammatory reaction involves various components of the immune system: B and T cells, macrophages, cytokines, antibodies and the complement system ${ }^{1}$. Immunoglobulins produced by activated B-lymphocytes are capable of crossing the brain-blood barrier. An elevation of the CSF IgG and the IgG index, and the presence of oligoclonal bands in the CSF are characteristics of MS. However; these findings are not exclusive to MS. OCB can be found in other inflammatory and chronic infections of the CNS. Only $72 \%$ of MS patients present with an elevated $\operatorname{IgG}$ index and $98 \%$ of MS patients have intrathecal OCB, which are not found in serum ${ }^{2,3}$.

The aim of our study was to determine whether there were any differences in intrathecal synthesis (IgG index) and the number of OCB among particular types of MS. The goal was to determine if intrathecal synthesis varies at the onset and in the various stages of MS.

\section{GROUP AND METHODS}

We evaluated 120 CSF samples from 29 CIS patients and 91 MS patients. The MS group consisted of 77 patients with relapsing-remitting MS (RR), 6 patients with the primary progressive course of the disease (PP) and 8 patients in secondary progression (SP); mean age = 42 years (range $=18$ to 70 years); 89 female and 31 males (Table 1). CSF evaluation in MS patients was part of the differential diagnostics process. All patients signed informed consent for a lumbar puncture. A total of $15 \mathrm{ml}$ of CSF was collected in glass tubes (via lumbar puncture using an atraumatic needle) and $15 \mathrm{ml}$ of blood were drawn from each patient. Within 30 min of collection, the CSF was centrifuged $\left(10 \mathrm{~min}, 1100 \mathrm{~g}, 4^{\circ} \mathrm{C}\right.$ ) and both serum and CSF were deep frozen $\left(-20^{\circ} \mathrm{C}\right)$ until analysis. The albumin and IgG in serum and CSF were determined in a certificated laboratory with a nephelometry using commercially available kits. The albumin quotient (CSF albumin / serum albumin) was used as a measure of the blood-brain barrier (BBB) integrity in conjunction with the $\mathrm{IgG}$ quotient (CSF IgG / serum IgG). The IgG index (IgG quotient / albumin quotient) was used for the determination of intrathecal synthesis. An IgG index value of $>0.7$ was taken to indicate the presence of intrathecal $\mathrm{IgG}$ synthesis. Oligoclonal $\mathrm{IgG}$ bands were assessed in both compartments using isoelectric focusing (IEF) on 
Table 1. Patient group characteristics.

\begin{tabular}{|c|c|c|c|c|c|c|}
\hline \multirow[t]{2}{*}{ Group } & \multicolumn{2}{|c|}{$\begin{array}{c}\text { Sex } \\
\text { (proportion) }\end{array}$} & \multirow[t]{2}{*}{ Total } & \multicolumn{2}{|c|}{$\begin{array}{l}\text { Mean age } \\
\text { (years) }\end{array}$} & \multirow[t]{2}{*}{ Overall (years) } \\
\hline & $\mathrm{F}$ & $\mathbf{M}$ & & $\mathrm{F}$ & M & \\
\hline \multirow[t]{2}{*}{ CIS } & 23 & 6 & 29 & 34.85 & 30.8 & 33.97 \\
\hline & $79.3 \%$ & $20.7 \%$ & & & & \\
\hline \multirow[t]{2}{*}{ RR } & 56 & 21 & 77 & 41.33 & 38.99 & 40.47 \\
\hline & $72.7 \%$ & $21.3 \%$ & & & & \\
\hline \multirow[t]{2}{*}{ SP } & 7 & 1 & 8 & 47.18 & 48.24 & 47.88 \\
\hline & $87.5 \%$ & $12.5 \%$ & & & & \\
\hline \multirow[t]{2}{*}{ PP } & 3 & 3 & 6 & 51.47 & 48.50 & 49.50 \\
\hline & $50.0 \%$ & $50.0 \%$ & & & & \\
\hline \multirow[t]{2}{*}{ Total } & 89 & 31 & 120 & 41.52 & 42.00 & 41.92 \\
\hline & $74.2 \%$ & $25.8 \%$ & & & & \\
\hline
\end{tabular}

Table 2. IgG indexes, OCB and EDSS in particular MS forms.

\begin{tabular}{llcccc}
\hline MS type & & CIS & RR & SP & PP \\
\hline Count & & 29 & 77 & 8 & 6 \\
\hline IgG index & median & 0.705 & 0.780 & 1.14 & 1.24 \\
& mean & 1.006 & 0.948 & 1.067 & 0.974 \\
& St. deviation & 0.549 & 0.377 & 0.367 & 0.375 \\
& $<0.7$ & $15(51.7 \%)$ & $24(31.2 \%)$ & $3(37.5 \%)$ & $2(33.3 \%)$ \\
& $>0.7$ & $14(49.3 \%)$ & $53(69.8 \%)$ & $5(63.5 \%)$ & $4(66.6 \%)$ \\
\hline OCB(count) & median & 7.0 & 8.0 & 8.0 & 12.5 \\
& mean & 10.1 & 8.97 & 8.88 & 13.0 \\
& St. deviation & 8.962 & 6.537 & 5.54 & 7.321 \\
& $<2$ & $3(10.3 \%)$ & $14(18.2 \%)$ & $1(12.5 \%)$ & $1(16.7 \%)$ \\
& $\geq 2$ & $26(89.7 \%)$ & $61(81.8 \%)$ & $7(87.5 \%)$ & $5(83.3 \%)$ \\
\hline EDSS & median & 1.0 & 2.0 & 6.0 & 4.75 \\
& mean & 0.79 & 2.01 & 5.75 & 4.83 \\
\end{tabular}

agarose gels followed by immunoblotting. OCB numbers $<2$ were considered negative.

The Kurtzke Expanded Disability Status Scale (EDSS) was determined for all participants before the lumbar puncture procedure.

Statistical analysis was completed using SPSS version 15 (SPSS Inc., Chicago, USA). Since the distribution values of evaluated parameters were not normal and there was an apparent presence of outlier values in the data, the non-parametric Kruskal-Wallis test was used for group comparisons. When the Kruskal-Wallis test was significant, the non-parametric Mann-Whitney test was subsequently used for post-hoc comparisons between pairs of evaluated groups. The chi-square test was applied to categorical data. A significance level of $P=0.05$ was used for all tests.

\section{RESULTS}

The CIS patients were significantly younger than the other evaluated groups ( $P=0.109$ to RRSM, $P=0.0032$ to
PP, $P=0.0030$ to SP, ANOVA and LSD post-hoc test). We found no statistically significant differences between the $\mathrm{IgG}$ index and the number of OCB in particular MS types. Only the means and medians of the OCB in PP were higher than in other MS types (mean in $\mathrm{PP}=13.0$, median $=12.5$; mean in CIS $=10.1$, median $=7.0$; mean in $\mathrm{RR}=8.97$, median=8.0; mean in $\mathrm{SP}=8.88$, median=8.0). EDSS in CIS patients was significantly lower than in the other groups. There was no correlation between EDSS values and intrathecal synthesis (IgG index; (QIgG / Qalbumin) and OCB.

\section{DISCUSSION}

The discovery of OCB in cerebrospinal fluid (CSF) was a major step forward in the understanding MS and other inflammatory diseases of the CNS. The key observation that led to the discovery of OCB dates to 1942, when Kabat described elevated gamma globulins in the CSF of MS patients. In 1959, Karcher, van Sande and Lowenthal reported that agar electrophoresis could differentiate CSF 
gamma-globulins from a patient with subacute sclerosing panencephalitis ${ }^{4,5}$. Since 1986, OCB detection in CSF has been routinely carried out using isoelectric focusing on agarose gel with immunoblotting. OCB assessment in the CSF is currently important in the diagnosis of MS; their presence supports this diagnosis if they are not found in the serum ${ }^{6}$. According to the revised McDonald criteria, the presence of OCB in CSF or IgG index elevation is one of the conditions for a diagnosis of MS ( ref. $^{7}$ ).

In our expectacion, there are not any differences in intrathecal synthesis represented by IgG index and OC bands numbers in the CNS after the first clinical manifestation of MS or in the course or stages of disease developed. The observation confirms the presence of inflammation in the CNS from the onset of disease and throughout the disease process. Higher numbers of OCB in CSF in our group of primary progressive patients was probably an anomaly, perhaps associated with sample size. Koch et al. did not find any differences in the presence or number of CSF OCB between patients with stable RRMS and SP patients experiencing significant exacerbation $^{8}$. In our work from 2008, we have yet to find any correlation between $\mathrm{IgG}$ index values and the number of OCB in the CSF of MS patients ${ }^{3}$. As we noted, there was no correlation between levels of disability, as measured using the EDSS, and intrathecal synthesis as an inflammatory marker. These results support the literature that the level of disability is caused by advancing neurodegeneration and axonal loss, but is not due to the intensity of inflammation. Similarly, CIS patients had a lower EDSS score due to their short history with the disease.

\section{CONCLUSION}

We found no statistically significant differences between the $\mathrm{IgG}$ index and the number of OCB in particular types of MS. There was no correlation between EDSS values and intrathecal synthesis (IgG index: (QIgG / Qalbumin)) and the number of OCB.

\section{ABBREVIATIONS}

CIS, Clinical isolated syndrome; CNS, Central nervous system; CSF, Cerebrospinal fluid; EDSS, Expanded Disability Status Scale; IEF, Isoelectric focusing; IgG, Immunoglobulin G; MS, Multiple sclerosis; OCB, Oligoclonal bands; PP, Primary progressive multiple sclerosis; RR, Relapsing - remitting multiple sclerosis; SP, Secondary progressive multiple sclerosis.

\section{ACKNOWLEDGEMENT}

This work was supported by the grant of the Ministry of Education no. MSM 6198959216.

\section{REFERENCES}

1. Kuenz B, Lutterotti Al., Ehling R, Gneiss C, Haemmerle M, Rainer C, Deisenhammer F, Schocke M, Berger T, Reindl M. Cerebrospinal fluid B Cells Correlate with Early Brain Inflammation in Multiple Sclerosis. Plos One 2008;3(7):2559.

2. Petereit H-F, Reske D. Expansion of antibody reactivity in the cerebrospinal fluid of multiple sclerosis patients - follow-up and clinical implications. Cerebrospin fluid Res 2005;2:3.

3. Mares J, Herzig R, Urbanek K, Sladkova V, Sklenarova J, Bekarek V, Schneiderka P, Zapletalova J, Kanovsky P. Correlation of the IgG index and oligoclonal bands in the cerebrospinal fluid of patiens with multiple sclerosis. Biomed Pap Med Fac Univ Palacky University Czech Rep. 2008,152(2):247-9.

4. HolmøyT. The Discovery of Oligoclonal Bands: A 50-Year Anniversary. Eur Neurol 2009;62:311-5.

5. Rammohan KW. Cerebrospinal fluid in multiple sclerosis. Ann Indian Acad Neurol 2009;12(4):246-53.

6. Freedman M, Thompson E, Deisenhammer F, Giovannoni G, Grimsley G, Keir G, Ohman S, Racke MK, Sharief M, Sindic CH, Sellebjerg F, Tourtellotte WV. Recommended Standart of Cerebrospinal Fluid Analysis in the Diagnosis of Multiple Sclerosis. Arch Neurol 2005;62:865-70.

7. Polman CH, Reingold SC, Edan G, Filippi M, Hartung HP, Kappos LI., Lublin FD, Metz LM, McFarland HF, O'Connor PW, Sandberg-Wollhein M, Thompson AJ, Weinshenker BG, Wolinsky JS. Diagnostic criteria for multiple sclerosis: 2005 revisions to the "McDonald criteria". Ann Neurol 2005;58:840-6.

8. Koch M, Heersema D, Mostert J, Teelken A, De Keyser J. Cerebrospinal fluid oligoclonal bands and progression of disability in multiple sclerosis. Eur J Neurol 2007;14(7):797-800. 